\title{
Dispersion analysis of the nucleon form factors including meson continua
}

\author{
M.A. Belushkin* and H.-W. Hamment \\ Helmholtz-Institut für Strahlen- und Kernphysik (Theorie), \\ Universität Bonn, Nußallee 14-16, \\ D-53115 Bonn, Germany \\ Ulf-G. Meißner \\ Helmholtz-Institut für Strahlen- und Kernphysik (Theorie), \\ Universität Bonn, Nußallee 14-16, \\ D-53115 Bonn, Germany \\ and \\ Institut für Kernphysik (Theorie), \\ Forschungszentrum Jülich, \\ D-52425 Jülich, Germany
}

(Dated: February 2, 2008)

\begin{abstract}
Dispersion relations provide a powerful tool to analyse the electromagnetic form factors of the nucleon for all momentum transfers. Constraints from meson-nucleon scattering data, unitarity, and perturbative QCD can be included in a straightforward way. In particular, we include the $2 \pi, \rho \pi$, and $K \bar{K}$ continua as independent input in our analysis and provide an error band for our results. Moreover, we discuss two different methods to include the asymptotic constraints from perturbative QCD. We simultaneously analyze the world data for all four form factors in both the space-like and time-like regions and generally find good agreement with the data. We also extract the nucleon radii and the $\omega N N$ coupling constants. For the radii, we generally find good agreement with other determinations with the exception of the electric charge radius of the proton which comes out smaller. The $\omega N N$ vector coupling constant is determined relatively well by the fits, but for the tensor coupling constant even the sign can not be determined.
\end{abstract}

PACS numbers: 11.55.Fv, 13.40.Gp, 14.20.Dh

*Electronic address: belushki@itkp.uni-bonn.de

${ }^{\dagger}$ Electronic address: hammer@itkp.uni-bonn.de

${ }^{\ddagger}$ Electronic address: meissner@itkp.uni-bonn.de 


\section{INTRODUCTION}

The electromagnetic (em) form factors of the nucleon provide an important tool to study strong interaction dynamics over a wide range of momentum transfers [1, 2]. Their detailed understanding is important for unraveling aspects of perturbative and nonperturbative nucleon structure. At small momentum transfers, they are determined by gross properties of the nucleon like the charge and magnetic moment. At high momentum transfers they encode information on the quark substructure of the nucleon as described by perturbative QCD. The form factors also contain important information on nucleon radii and vector meson coupling constants. Moreover, they are an important ingredient in a wide range of experiments from Lamb shift measurements [3] to determinations of the strangeness content of the nucleon [4].

With the advent of the new continuous beam electron accelerators such as CEBAF (Jefferson Laboratory), ELSA (Bonn), and MAMI (Mainz), a wealth of precise data for space-like momentum transfers has become available [5]. Due to the difficulty of the experiments, the time-like form factors are less well known. While there is a fair amount of information on the proton time-like form factors 6, 7], only one measurement of the neutron form factor from the pioneering FENICE experiment [8, 9] exists. Recently, new precise data on the proton time-like form factors have been presented by the BES, CLEO, and BABAR collaborations [10, 11, 12].

In this work, we therefore analyze the nucleon form factors in both the space- and timelike regions. We use dispersion relations which provide a model-independent framework to consistently analyze the form factor data in both regions. A complete description of our data base is given in section $\nabla$

It has been known for a long time that the pion plays an important role in the longrange structure of the nucleon [13]. This connection was made more precise using dispersion theory in the 1950's 14, 15. Subsequently, Frazer and Fulco have written down partial wave dispersion relations that relate the nucleon electromagnetic structure to pion-nucleon $(\pi N)$ scattering and predicted the existence of the $\rho$ resonance [16, 17]. Höhler et al. [18] first performed a dispersion analysis of the electromagnetic form factors of the nucleon including the $2 \pi$ continuum derived from the pion form factor and $\pi N$-scattering data 19]. In the mid-nineties, this analysis has been updated by Mergell, Meißner, and Drechsel [20] and was later extended to include data in the time-like region [21, 22]. The new precise data for the neutron electric form factor have been included as well [23]. For recent attempts to calculate the nucleon form factors in QCD using nonperturbative methods, see e.g. Refs. [24, 25, 26].

A recent form factor analysis by Friedrich and Walcher 27] created some renewed interest in the $2 \pi$ continuum. They analysed the electromagnetic nucleon form factors and performed various phenomenological fits [27]. Their fits showed a pronounced bump-dip structure in $G_{E}^{n}$ which they interpreted as a signature of a very long-range contribution of the pion cloud to the charge distribution in the Breit frame extending out to about $2 \mathrm{fm}$. This observation is at variance with the pion cloud contribution to the nucleon form factors as given by the $2 \pi$ continuum - the lowest-mass intermediate state including pions only [28]. We will discuss a possibility how to reconcile these findings below.

It is well known that vector mesons play an important role in the electromagnetic structure of the nucleon, see e.g. Refs. [16, 29, 30, 31, 32, 33, 34], and the remaining contributions to the spectral function have usually been approximated by vector meson resonances. A novel addition in this work is the inclusion of the $K \bar{K}$ [35, 36] and $\rho \pi$ [37] continuum contributions similar to the $2 \pi$ continuum described above. The continuum contributions provide 
independent information on the spectral functions that supplements the electromagnetic form factor data. Moreover, we enforce the asymptotic constraints from pQCD. We discuss two different approaches to obtain this behavior: superconvergence relations and an explicit continuum term with the correct pQCD behavior, and show results for both methods.

Our manuscript is organized as follows. Sec. \A contains the basic definitions of the nucleon em form factors and a short discussion of the corresponding dispersion relations. The various continuum contributions to the spectral functions are discussed in Sec. IIIA. The structure of and the constraints on the spectral functions are given in Sec. IV. The results of our fits are presented and discussed in Sec V. We close with a short summary and outlook in Sec. VI. The fit parameters are collected in the appendix.

\section{PRELIMINARIES}

\section{A. Definitions}

The electromagnetic (em) structure of the nucleon is determined by the matrix element of the vector current operator $j_{\mu}^{\mathrm{em}}$ between nucleon states as illustrated in Fig. 1.

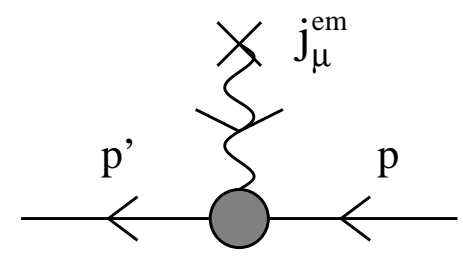

FIG. 1: The nucleon matrix element of the electromagnetic current $j_{\mu}^{\mathrm{em}}$.

Using Lorentz and gauge invariance, this matrix element can be expressed in terms of two form factors,

$$
\left\langle p^{\prime}\left|j_{\mu}^{\mathrm{em}}\right| p\right\rangle=\bar{u}\left(p^{\prime}\right)\left[F_{1}(t) \gamma_{\mu}+i \frac{F_{2}(t)}{2 M} \sigma_{\mu \nu} q^{\nu}\right] u(p),
$$

where $M$ is the nucleon mass and $t=\left(p^{\prime}-p\right)^{2}$ the four-momentum transfer squared. For data in the space-like region, it is often convenient to use the variable $Q^{2}=-t>0$. The functions $F_{1}(t)$ and $F_{2}(t)$ are the Dirac and Pauli form factors, respectively. They are normalized at $t=0$ as

$$
F_{1}^{p}(0)=1, F_{1}^{n}(0)=0, F_{2}^{p}(0)=\kappa_{p}, F_{2}^{n}(0)=\kappa_{n},
$$

with $\kappa_{p}=1.793$ and $\kappa_{n}=-1.913$ the anomalous magnetic moment of the proton and the neutron, respectively, in units of nuclear magnetons.

It is convenient to work in the isospin basis and to decompose the form factors into isoscalar and isovector parts,

$$
F_{i}^{s}=\frac{1}{2}\left(F_{i}^{p}+F_{i}^{n}\right), \quad F_{i}^{v}=\frac{1}{2}\left(F_{i}^{p}-F_{i}^{n}\right),
$$

where $i=1,2$. The experimental data are usually given for the Sachs form factors

$$
\begin{aligned}
G_{E}(t) & =F_{1}(t)-\tau F_{2}(t), \\
G_{M}(t) & =F_{1}(t)+F_{2}(t),
\end{aligned}
$$


where $\tau=-t /\left(4 M^{2}\right)$. In the Breit frame, $G_{E}$ and $G_{M}$ may be interpreted as the Fourier transforms of the charge and magnetization distributions, respectively.

The nucleon radii $\sqrt{\left\langle r^{2}\right\rangle}$ can be defined from the low- $t$ expansion of the form factors,

$$
F(t)=F(0)\left[1+t\left\langle r^{2}\right\rangle / 6+\ldots\right]
$$

where $F(t)$ is a generic form factor. In the case of the electric and Dirac form factors of the neutron, $G_{E}^{n}$ and $F_{1}^{n}$, the expansion starts with the term linear in $t$ and the normalization factor $F(0)$ is dropped.

\section{B. Dispersion Relations and Spectral Decomposition}

Based on unitarity and analyticity, dispersion relations relate the real and imaginary parts of the electromagnetic nucleon form factors. Let $F(t)$ be a generic symbol for any one of the four independent nucleon form factors. We write down an unsubtracted dispersion relation of the form

$$
F(t)=\frac{1}{\pi} \int_{t_{0}}^{\infty} \frac{\operatorname{Im} F\left(t^{\prime}\right)}{t^{\prime}-t-i \epsilon} d t^{\prime}
$$

where $t_{0}$ is the threshold of the lowest cut of $F(t)$ (see below) and the $i \epsilon$ defines the integral for values of $t$ on the cut. The convergence of an unsubtracted dispersion relation for the form factors has been assumed. We could also use a once subtracted dispersion relation, since the normalization of the form factors at $t=0$ is known. Using Eq. (6) the electromagnetic structure of the nucleon can be related to its absorptive behavior.

The imaginary part $\operatorname{Im} F$ entering Eq. (6) can be obtained from a spectral decomposition [14, 15]. For this purpose it is most convenient to consider the electromagnetic current matrix element in the time-like region $(t>0)$, which is related to the space-like region $(t<0)$ via crossing symmetry. The matrix element can be expressed as

$$
\begin{aligned}
J_{\mu} & =\left\langle N(p) \bar{N}(\bar{p})\left|j_{\mu}^{\mathrm{em}}(0)\right| 0\right\rangle \\
& =\bar{u}(p)\left[F_{1}(t) \gamma_{\mu}+i \frac{F_{2}(t)}{2 M} \sigma_{\mu \nu}(p+\bar{p})^{\nu}\right] v(\bar{p}),
\end{aligned}
$$

where $p$ and $\bar{p}$ are the momenta of the nucleon and antinucleon created by the current $j_{\mu}^{\mathrm{em}}$, respectively. The four-momentum transfer squared in the time-like region is $t=(p+\bar{p})^{2}$.

Using the LSZ reduction formalism, the imaginary part of the form factors is obtained by inserting a complete set of intermediate states as [14, 15]

$$
\operatorname{Im} J_{\mu}=\frac{\pi}{Z}(2 \pi)^{3 / 2} \mathcal{N} \sum_{\lambda}\left\langle p\left|\bar{J}_{N}(0)\right| \lambda\right\rangle\left\langle\lambda\left|j_{\mu}^{\mathrm{em}}(0)\right| 0\right\rangle v(\bar{p}) \delta^{4}\left(p+\bar{p}-p_{\lambda}\right),
$$

where $\mathcal{N}$ is a nucleon spinor normalization factor, $Z$ is the nucleon wave function renormalization, and $\bar{J}_{N}(x)=J^{\dagger}(x) \gamma_{0}$ with $J_{N}(x)$ a nucleon source. This decomposition is illustrated in Fig. 2. It relates the spectral function to on-shell matrix elements of other processes.

The states $|\lambda\rangle$ are asymptotic states of momentum $p_{\lambda}$. They carry the same quantum numbers as the current $j_{\mu}^{\mathrm{em}}: I^{G}\left(J^{P C}\right)=0^{-}\left(1^{--}\right)$for the isoscalar current and $I^{G}\left(J^{P C}\right)=$ $1^{+}\left(1^{--}\right)$for the isovector component of $j_{\mu}^{\mathrm{em}}$. Furthermore, they have zero net baryon number. Because of $G$-parity, states with an odd number of pions only contribute to the isoscalar 


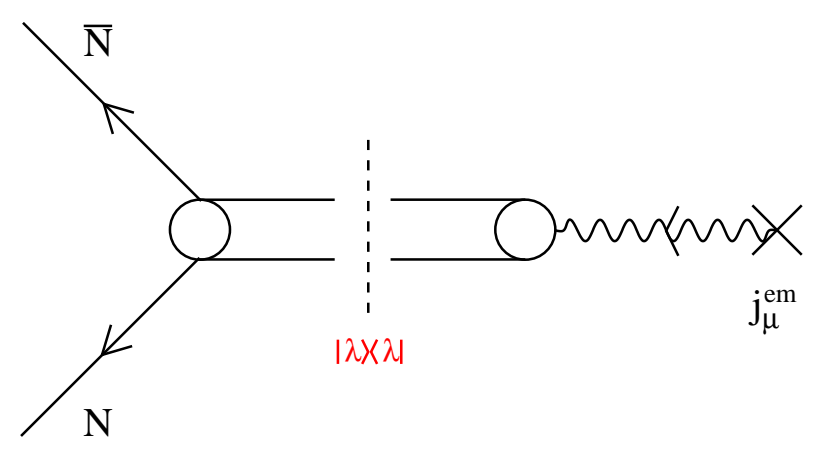

FIG. 2: The spectral decomposition of the nucleon matrix element of the electromagnetic current $j_{\mu}^{\mathrm{em}} .|\lambda\rangle$ denotes an hadronic intermediate state.

part, while states with an even number contribute to the isovector part. For the isoscalar part the lowest mass states are: $3 \pi, 5 \pi, \ldots, K \bar{K}, K \bar{K} \pi, \ldots$; for the isovector part they are: $2 \pi, 4 \pi, \ldots$.

Associated with each intermediate state is a cut starting at the corresponding threshold in $t$ and running to infinity. As a consequence, the spectral function $\operatorname{Im} F(t)$ is different from zero along the cut from $t_{0}$ to $\infty$, with $t_{0}=4(9) M_{\pi}^{2}$ for the isovector (isoscalar) case.

The spectral functions are the central quantities in the dispersion-theoretical approach. Using Eqs. (78), they can in principle be constructed from experimental data. In practice, this program can only be carried out for the lightest two-particle intermediate states.

The longest-range, and therefore at low momentum transfer most important continuum contribution comes from the $2 \pi$ intermediate state which contributes to the isovector form factors [19]. A new calculation of this contribution has recently been performed in Ref. [38]. In this analysis we for the first time also include the $K \bar{K}$ and $\rho \pi$ continua [35, 36, 37]. ${ }^{1}$

\section{CONTINUUM CONTRIBUTIONS}

Our general strategy is to include as much physics information in the construction of the spectral functions as possible. In this section, we explicitly contruct the $2 \pi, K \bar{K}$, and $\rho \pi$ continua mentioned above. These continua will be an important part of our spectral functions.

\section{A. $2 \pi$ Continuum}

The $2 \pi$ contribution has recently been reevaluated in a model-independent way 38] using the latest experimental data for the pion form factor from CMD-2 [39], KLOE [40], and SND [41]. Here we give a short summary of this evaluation.

Following Refs. [38, 42], the $2 \pi$ contribution to the isovector spectral functions in terms of the pion charge form factor $F_{\pi}(t)$ and the P-wave $\pi \pi \rightarrow \bar{N} N$ amplitudes $f_{ \pm}^{1}(t)$ can be

\footnotetext{
${ }^{1}$ Note that the effect of these continua has previously been studied in Ref. [36] using fits to parameterizations of form factor data.
} 
expressed as:

$$
\begin{aligned}
\operatorname{Im} G_{E}^{v}(t) & =\frac{q_{t}^{3}}{M \sqrt{t}} F_{\pi}(t)^{*} f_{+}^{1}(t), \\
\operatorname{Im} G_{M}^{v}(t) & =\frac{q_{t}^{3}}{\sqrt{2 t}} F_{\pi}(t)^{*} f_{-}^{1}(t),
\end{aligned}
$$

where $q_{t}=\sqrt{t / 4-M_{\pi}^{2}}$. The imaginary parts of the Dirac and Pauli Form factors can be obtained using Eq. (4). The $2 \pi$ continuum is expected to be the dominant contribution to the isovector spectral function from threshold up to masses of about $\sqrt{t} \approx 1 \mathrm{GeV}[42$.

The $\mathrm{P}$-wave $\pi \pi \rightarrow \bar{N} N$ amplitudes $f_{ \pm}^{1}(t)$ are tabulated in Ref. [42]. The representation of Eq. (9) gives the exact isovector spectral functions for $4 M_{\pi}^{2} \leq t \leq 16 M_{\pi}^{2}$, but in practice holds up to $t \simeq 50 M_{\pi}^{2} \approx 1 \mathrm{GeV}^{2}$. Since the contributions from $4 \pi$ and higher intermediate states is small up to $t \simeq 50 M_{\pi}^{2}, F_{\pi}(t)$ and the $f_{ \pm}^{1}(t)$ share the same phase in this region and the two quantities can be replaced by their absolute values.

The experimental data for the pion form factor from CMD-2 39], KLOE [40], and SND 41] show some discrepancies. In Ref. 38], the $2 \pi$ continuum given by Eq. (9) was evaluated for all three sets and the errors from the discrepancy between the sets were estimated. The resulting difference in the spectral functions is very small $(\lesssim 1 \%)$. It is largest in the $\rho$-peak region, but this region is suppressed by the $\pi \pi \rightarrow \bar{N} N$ amplitudes $f_{ \pm}^{1}(t)$ which show a strong fall-off as $t$ increases.

The spectral functions have two distinct features. First, as already pointed out in [16], they contain the important contribution of the $\rho$-meson with its peak at $t \simeq 30 M_{\pi}^{2}$. Second, on the left shoulder of the $\rho$, the isovector spectral functions display a very pronounced enhancement close to the two-pion threshold. This is due to the logarithmic singularity on the second Riemann sheet located at $t_{c}=4 M_{\pi}^{2}-M_{\pi}^{4} / M^{2}=3.98 M_{\pi}^{2}$, very close to the threshold. If one were to neglect this important unitarity correction, one would severely underestimate the nucleon isovector radii [43]. In fact, precisely the same effect is obtained at leading one-loop accuracy in relativistic chiral perturbation theory 44, 45]. This topic was also discussed in heavy baryon chiral perturbation theory (ChPT) [46, 47] and in a covariant calculation based on infrared regularization [48, 49]. Thus, the most important $2 \pi$ contribution to the nucleon form factors can be determined by using either unitarity or ChPT (in the latter case, of course, the $\rho$ contribution is not included).

The contribution to the nucleon form factors is obtained by inserting the $2 \pi$ contribution to the spectral function into the dispersion relations Eq. (6). The result can be parameterized as

$$
F_{i}^{(v, 2 \pi)}(t)=\frac{a_{i}+b_{i}\left(1-t / c_{i}\right)^{-2 / i}}{2\left(1-t / d_{i}\right)}, \quad i=1,2,
$$

where $a_{1}=1.10788, b_{1}=0.109364, c_{1}=0.36963 \mathrm{GeV}^{2}, d_{1}=0.553034 \mathrm{GeV}^{2}, a_{2}=5.724253$, $b_{2}=1.111128, c_{2}=0.27175 \mathrm{GeV}^{2}$, and $d_{2}=0.611258 \mathrm{GeV}^{2}$. The errors in these constants are of the order $4 \%$ or less. Of course, it is not necessary to use a parameterization like Eq. (10) but the numerical evaluation of the fits becomes much simpler. The form factor contributions from Eq. (10) are shown in Fig. 3 below. 


\section{B. $K \bar{K}$ Continuum}

The $K \bar{K}$ contribution to the isoscalar spectral function was evaluated in Refs. [19, 35, 36 ] from an analytic continuation of $K N$ scattering data. In the following, we give a short summary of this work.

The $K \bar{K}$ contribution to the imaginary part of the isocalar form factors is given by [35, 36]

$$
\begin{aligned}
& \operatorname{Im} F_{1}^{(s, K \bar{K})}(t)=\operatorname{Re}\left\{\left(\frac{M q_{t}}{4 p_{t}^{2}}\right)\left[\frac{\sqrt{t}}{2 \sqrt{2} M} b_{1}^{1 / 2,-1 / 2}(t)-b_{1}^{1 / 2,1 / 2}(t)\right] F_{K}(t)^{*}\right\}, \\
& \operatorname{Im} F_{2}^{(s, K \bar{K})}(t)=\operatorname{Re}\left\{\left(\frac{M q_{t}}{4 p_{t}^{2}}\right)\left[b_{1}^{1 / 2,1 / 2}(t)-\frac{\sqrt{2} M}{\sqrt{t}} b_{1}^{1 / 2,-1 / 2}(t)\right] F_{K}(t)^{*}\right\},
\end{aligned}
$$

with $p_{t}=\sqrt{t / 4-M^{2}}$ and $q_{t}=\sqrt{t / 4-M_{K}^{2}} \cdot F_{K}(t)$ represents the kaon form factor whereas the $b_{1}^{1 / 2, \pm 1 / 2}(t)$ are the $J=1$ partial wave amplitudes for $K \bar{K} \rightarrow N \bar{N}$ [35, 36]. Once these imaginary parts are determined, the contribution of the $K \bar{K}$-continuum to the form factors is obtained from the dispersion relation Eq. (6)).

For $t \geq 4 M^{2}$ the partial waves are bounded by unitarity,

$$
\sqrt{p_{t} / q_{t}}\left|b_{1}^{1 / 2, \pm 1 / 2}(t)\right| \leq 1 \text {. }
$$

In the unphysical region $4 M_{K}^{2} \leq t \leq 4 M^{2}$, however, they are not constrained by unitarity. In Ref. [35], the amplitudes $b_{1}^{1 / 2, \pm 1 / 2}(t)$ in the unphysical region have been determined from an analytic continuation of $K N$-scattering amplitudes. The contribution of the physical region $t \geq 4 M^{2}$ in the dispersion integral (6) is suppressed for small momentum transfers and bounded because of Eq. (13). Using the analytically continued amplitudes in the unphysical region and the unitarity bound in the physical region, the contribution of the $K \bar{K}$ continuum can therefore be calculated. Strictly speaking this calculation provides an upper bound on the spectral function since we replace the amplitudes and the form factor in Eqs. (11), 121) by their absolute values.

The striking feature in the spectral function is a clear $\phi$ resonance structure just above the $K \bar{K}$ threshold. The resonance structure appears in the partial wave amplitude $b_{1}^{1 / 2,1 / 2}$ as well as in the kaon form factor $F_{K}$. In contrast to the $2 \pi$ continuum, there is no strong enhancement on the left wing of the $\phi$ resonance which sits directly at the $K \bar{K}$ threshold.

The resulting contribution to the nucleon form factors can be parameterized by a pole term at the $\phi$ mass:

$$
F_{i}^{(s, K \bar{K})}(t)=\frac{1}{\pi} \int_{4 M_{K}^{2}}^{\infty} \frac{\operatorname{Im} F_{i}^{(s, K \bar{K})}\left(t^{\prime}\right)}{t^{\prime}-t} d t^{\prime} \approx \frac{a_{i}^{K \bar{K}}}{M_{\phi}^{2}-t}, \quad i=1,2,
$$

with $a_{1}^{K \bar{K}}=0.1054 \mathrm{GeV}^{2}$ and $a_{2}^{K \bar{K}}=0.2284 \mathrm{GeV}^{2}$. As a consequence, the contribution of the $K \bar{K}$ continuum to the electromagnetic nucleon form factors can conveniently be included in the analysis via Eq. (14). The form factor contributions from Eq. (14) are also shown in Fig. [3 below.

\section{C. $\rho \pi$ Continuum}

Drawing upon a realistic treatment of the correlated $\rho \pi$ exchange in the Bonn-Jülich $N N$ model [50], the $\rho \pi$ contribution to the isoscalar spectral function was calculated in Ref. [37]. 
The contribution of the $\rho \pi$ continuum can be evaluated in terms of a dispersion integral which in turn can be represented by an effective pole term for a fictitious $\omega^{\prime}$ meson with a $\operatorname{mass} M_{\omega^{\prime}}=1.12 \mathrm{GeV}[37]$ :

$$
F_{i}^{(s, \rho \pi)}(t)=\frac{1}{\pi} \int_{\left(M_{\pi}+M_{\rho}\right)^{2}}^{\infty} \frac{\operatorname{Im} F_{i}^{(s, \rho \pi)}\left(t^{\prime}\right)}{t^{\prime}-t} d t^{\prime} \approx \frac{a_{i}^{\rho \pi}}{M_{\omega^{\prime}}^{2}-t}, \quad i=1,2
$$

with $a_{1}^{\rho \pi}=-1.01 \mathrm{GeV}^{2}$ and $a_{2}^{\rho \pi}=-0.04 \mathrm{GeV}^{2}$. In our form factor analysis, we use this effective pole instead of the full spectral function.

There is very little sensitivity in our fits to $a_{2}^{\rho \pi}$, which can vary between -0.04 and -0.4 without affecting the outcome of the fit. If the $\omega^{\prime}$ pole is treated as a real resonance, the latter value is consistent with $f_{\omega^{\prime}} \sim 10$ for $a_{1}^{\rho \pi}=-1.01$ if the coupling constants $g_{\omega^{\prime} N N}^{i}$ $(i=1,2)$ from Ref. [37] are used as input.

In Fig. 3, we show the contribution of the $2 \pi, K \bar{K}$, and $\rho \pi$ continua to the electromagnetic
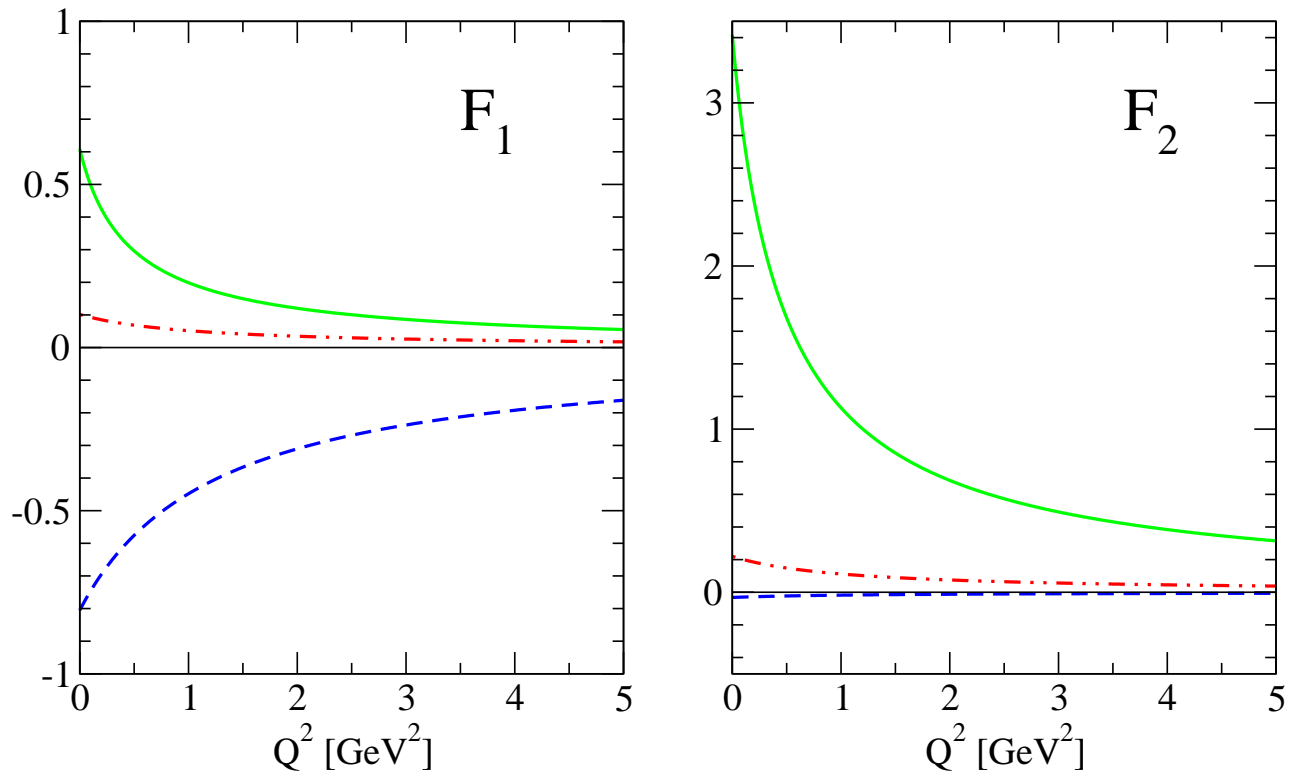

FIG. 3: The continuum contributions to the nucleon form factors $F_{1}$ (left panel) and $F_{2}$ (right panel) in the space-like region. The contribution of the $2 \pi$ continuum to the isovector form factors is given by the solid line, while the contribution of the $K \bar{K}$ and $\rho \pi$ continua to the isoscalar form factors are given by the dash-dotted and dashed lines, respectively.

nucleon form factors $F_{1}$ and $F_{2}$. The $2 \pi$ contributes to the isovector form factors while the $K \bar{K}$ and $\rho \pi$ continua contribute to the isoscalar form factors. The $K \bar{K}$ and $\rho \pi$ contributions have opposite sign and partially cancel each other. The dominant contribution to $F_{1}^{s}$ comes from the $\rho \pi$ continuum while for $F_{2}^{s}$ the $K \bar{K}$ contribution is larger. While the $K \bar{K}$ and $\rho \pi$ contributions can be represented by simple pole terms, the expressions for the $2 \pi$ continuum Eq. (10) are somewhat more complicated. This is related to the strong enhancement close to the $2 \pi$ threshold on the left wing of the $\rho$ resonance discussed above. Finally, note that these continuum contributions enter as an independent input in our analysis. They are not fitted to the form factor data. 


\section{SPECTRAL FUNCTIONS}

\section{A. Structure}

As discussed above, the spectral function can at present only be obtained from unitarity arguments and experimental data for the lightest two-particle intermediate states $(2 \pi$ and $K \bar{K})[19$, 35, 36]. The $\rho \pi$ continuum contribution has been calculated in the Bonn-Jülich $N N$ model [37].

The remaining contributions to the spectral function can be parameterized by vector meson poles. On one hand, the lower mass poles can be identified with physical vector mesons such as the $\omega$ and the $\phi$. In the the case of the $3 \pi$ continuum, e.g., it has been shown in ChPT that the nonresonant contribution is very small and the spectral function is dominated by the $\omega$ [46]. The higher mass poles on the other hand, are simply an effective way to parameterize higher mass strength in the spectral function. Different parameterizations are possible and an explicit example will be discussed below in relation to the pQCD behavior.

In all our fits the spectral function includes the $2 \pi, K \bar{K}$, and $\rho \pi$ continua from unitarity and the $\omega$ pole. Note that we also include a pole at the $\phi$ mass to account for explicit $\phi$ strength not included in the $K \bar{K}$ and $\rho \pi$ continua. In addition to that there are a number of effective poles at higher momentum transfers in the isoscalar $\left(s_{1}, s_{2}, \ldots\right)$ and isovector channels $\left(v_{1}, v_{2}, \ldots\right)$. The spectral function has the general structure

$$
\begin{aligned}
& \operatorname{Im} F_{i}^{s}(t)=\operatorname{Im} F_{i}^{(s, K \bar{K})}(t)+\operatorname{Im} F_{i}^{(s, \rho \pi)}(t)+\sum_{V=\omega, \phi, s_{1}, \ldots} \pi a_{i}^{V} \delta\left(M_{V}^{2}-t\right), \quad i=1,2, \\
& \operatorname{Im} F_{i}^{v}(t)=\operatorname{Im} F_{i}^{(v, 2 \pi)}(t)+\sum_{V=v_{1}, \ldots} \pi a_{i}^{V} \delta\left(M_{V}^{2}-t\right), \quad i=1,2 .
\end{aligned}
$$

The masses of the effective poles are fitted to the form factor data. We generally do not include widths for the effective poles. However, in some of the fits we allow a large width for the highest mass effective pole in order to mimick the imaginary part of the form factors in the time-like region. We have performed various fits with different numbers of effective poles and including/excluding some of the continuum contributions. In Sec. V we will discuss the results of these efforts.

\section{B. Constraints}

The number of parameters in the fit function is reduced by enforcing various constraints. The first set of constraints concerns the low- $t$ behavior of the form factors: We enforce the correct normalization of the form factors as given in Eq. (2). The nucleon radii, however, are not included as a constraint. In some earlier fits, we had also constrained the neutron charge radius to the value from low-energy neutron-atom scattering experiments [51, 52]. In the fits discussed below, this constraint is dropped since the fit value is compatible with the empirical range from Refs. 51, 52].

Perturbative QCD (pQCD) constrains the behavior of the nucleon electromagnetic form factors for large momentum transfer. Brodsky and Lepage [53] find for $Q^{2} \rightarrow \infty$,

$$
F_{i}(t) \rightarrow \frac{1}{Q^{2(i+1)}}\left[\ln \left(\frac{Q^{2}}{Q_{0}^{2}}\right)\right]^{-\gamma}, \quad i=1,2,
$$


where $Q_{0} \simeq \Lambda_{\mathrm{QCD}}$. The anomalous dimension $\gamma \approx 2$ depends weakly on the number of flavors [53]. The asymptotic behavior of the form factors has recently also been studied in connection to the unexpected behavior of the ratio $Q^{2} F_{2}\left(Q^{2}\right) / F_{1}\left(Q^{2}\right)$ for the proton measured at Jefferson Lab and different expressions for the logarithmic corrections were found [54, 55]. (For a further discussion of the asymptotic behavior of the nucleon form factors for large space-like and time-like momenta, see Ref. [56].) In the current analysis we implement only the leading power behavior of the form factors and details of the logarithmic correction are not relevant. Note that the logarithmic term in Eq. (18) was included in some of our earlier analyses [20, 21, 23] but had little impact on the fit. The particular way this constraint was implemented, however, lead to an unphysical logarithmic singularity of the form factors in the time-like region which we want to avoid in the current analysis.

The power behavior of the form factors at large $t$ can be easily understood from perturbative gluon exchange. In order to distribute the momentum transfer from the virtual photon to all three quarks in the nucleon, at least two massless gluons have to be exchanged. Since each of the gluons has a propagator $\sim 1 / t$, the form factor has to fall off as $1 / t^{2}$. In the case of $F_{2}$, there is additional suppression by $1 / t$ since a quark spin has to be flipped. The power behavior of the form factors leads to superconvergence relations of the form

$$
\int_{t_{0}}^{\infty} \operatorname{Im} F_{i}(t) t^{n} d t=0, \quad i=1,2
$$

with $n=0$ for $F_{1}$ and $n=0,1$ for $F_{2}$.

The pQCD power behavior can be enforced in various ways. To obtain some information about the induced theoretical uncertainty, we discuss two different methods in more detail:

1. Superconvergence (SC) approach:

The asymptotic behavior of Eq. (18) is obtained by choosing the residues of the vector meson pole terms such that the leading terms in the $1 / t$-expansion cancel. This leads to a spectral function consistent with the superconvergence relations Eq. (19) and the asymptotic behavior Eq. (18). This method is similar to what was used in earlier works [20, 21, 23]. Here we add a very broad resonance of the structure

$$
F_{i}^{(I, b r o a d)}(t)=\frac{a_{i}^{I}\left(M_{I}^{2}-t\right)}{\left(M_{I}^{2}-t\right)^{2}+\Gamma_{I}^{2}}, \quad i=1,2, \quad I=s, v
$$

in both the isovector and isoscalar form factors. The resonance parameterises continuum contributions in addition to the $2 \pi, K \bar{K}$, and $\rho \pi$ continua and generates an imaginary part of the form factors in the time-like region for $t \geq 4 M^{2}$. The residue $a_{i}^{I}$ as well as the mass and width parameters $M_{I}$ and $\Gamma_{I}$ are fit to the data. The width parameter $\Gamma_{I}$ is of the same order of magnitude as the mass $M_{I}$ and comes out typically of the order of a few $\mathrm{GeV}$ in our fits.

2. Explicit pQCD continuum approach:

In addition to satisfying the superconvergence relations, Eq. (19), a term of the form

$$
F_{i}^{(I, p Q C D)}=\frac{a_{i}^{I}}{1-c_{i}^{2} t+b_{i}^{2}(-t)^{i+1}}, \quad i=1,2, \quad I=s, v,
$$

which explicitly enforces the pQCD behavior, Eq. (18), is added to the fit function. Such a term behaves like an effective resonance pole for small values of $t$, and restores 
pQCD behaviour explicitly at high values of $t$. The superconvergence relations cancel the leading order terms in the $1 / t$ expansion. This explicit pQCD term is consistent with a nonvanishing imaginary part of the form factors in the time-like region. Note that the parameters $b_{i}$ and $c_{i}$ are the same in the isoscalar and isovector channels while the residue $a_{i}^{I}$ depends on the channel. This method allows for a smoother interpolation between the low- $t$ and large- $t$ regions compared to the SC approach. Because of this feature, one might expect obtaining fits with fewer parameters.

The number of effective poles in Eqs. (16, 17) is determined by the stability criterion discussed in detail in [57]. In short, we take the minimum number of poles necessary to fit the data. The number of free parameters is strongly reduced by the various constraints (unitarity, normalizations, superconvergence relations). More details will be given together with the fits in the next section.

\section{FIT RESULTS}

The fits have been performed using the Fletcher-Reeves and the Polak-Ribiere conjugate gradient algorithms implemented in the GSL library [58]. To ensure initial convergence stability, Monte-Carlo sampling was performed over the whole physically acceptable parametric volume to obtain a number of parameter sets with acceptable starting $\chi^{2}$ values.

The constraints dictated by the normalization and the pQCD conditions have been represented in terms of a set of linear equations for the resonance residua. The equations are solved each iteration using the LU decomposition.

Soft constraints on composite variables which depend on a set of fit parameters allow to impose an exponential well for the set of parameters as a whole, limiting deviations of the composite variable from its desired central value. These constraints are implemented as additive $\chi^{2}$ terms of the general form

$$
\tilde{\chi}^{2}=p[x-\langle x\rangle]^{2} \exp \left(p[x-\langle x\rangle]^{2}\right)
$$

where $\langle x\rangle$ is the desired central value, and $p$ is the constraint strength parameter which allows to stabilize fit convergence over the whole range of iterations adaptively, regulating the steepness of the exponential well. This method was used in some earlier fits to constrain the neutron radius [59].

The error bands are obtained by allowing the total $\chi^{2} /$ dof of the fit to be in the interval $\left[\chi_{\min }^{2}, \chi_{\min }^{2}+\delta \chi^{2}\right]$ where $\chi_{\min }^{2}$ is the $\chi^{2}$ value of the best fit, and $\delta \chi^{2}$ is obtained from the $1 \sigma$ confidence interval $p$-value equations, $\delta \chi^{2} \simeq 1.04$.

The data basis used in the fits is taken from Ref. [27] and in addition also includes the new data that have appeared since 2003 and the time-like data [7, 8, 9, 10, 11, 12, 60, 61, 62, 63, 64, 65, 66, 67, 68, 69, 70]. The CLAS collaboration at Jefferson Lab has performed measurements of the neutron magnetic form factor for momentum transfers $0.6 \leq Q^{2} \leq$ $5 \mathrm{GeV}^{2}[71$. These data are still preliminary and are therefore generally not included in our fits. In subsection $\mathrm{VD}$, however, we discuss a fit where these preliminary data are included. The results for $G_{M}^{n}, G_{E}^{p}, G_{M}^{p}$ are normalized to the phenomenological dipole fit:

$$
G_{D}\left(Q^{2}\right)=\left(1+\frac{Q^{2}}{m_{D}^{2}}\right)^{-2}
$$


where $m_{D}^{2}=0.71 \mathrm{GeV}^{2}$.

Additionally, certain features of the form factor behaviour in specific $Q^{2}$ ranges can be enhanced during the fitting procedure by artificially decreasing the errors on the experimental data in that region as seen by the fit. This allows to, for example, explore the conditions necessary to produce a pronounced bump-dip structure in $G_{E}^{n}$, discussed in Sec. VE.

In the time-like region, the neutron data do not participate in the fit as they are obtained from a single experiment. They are therefore a genuine prediction.

\section{A. Superconvergence (SC) Approach}
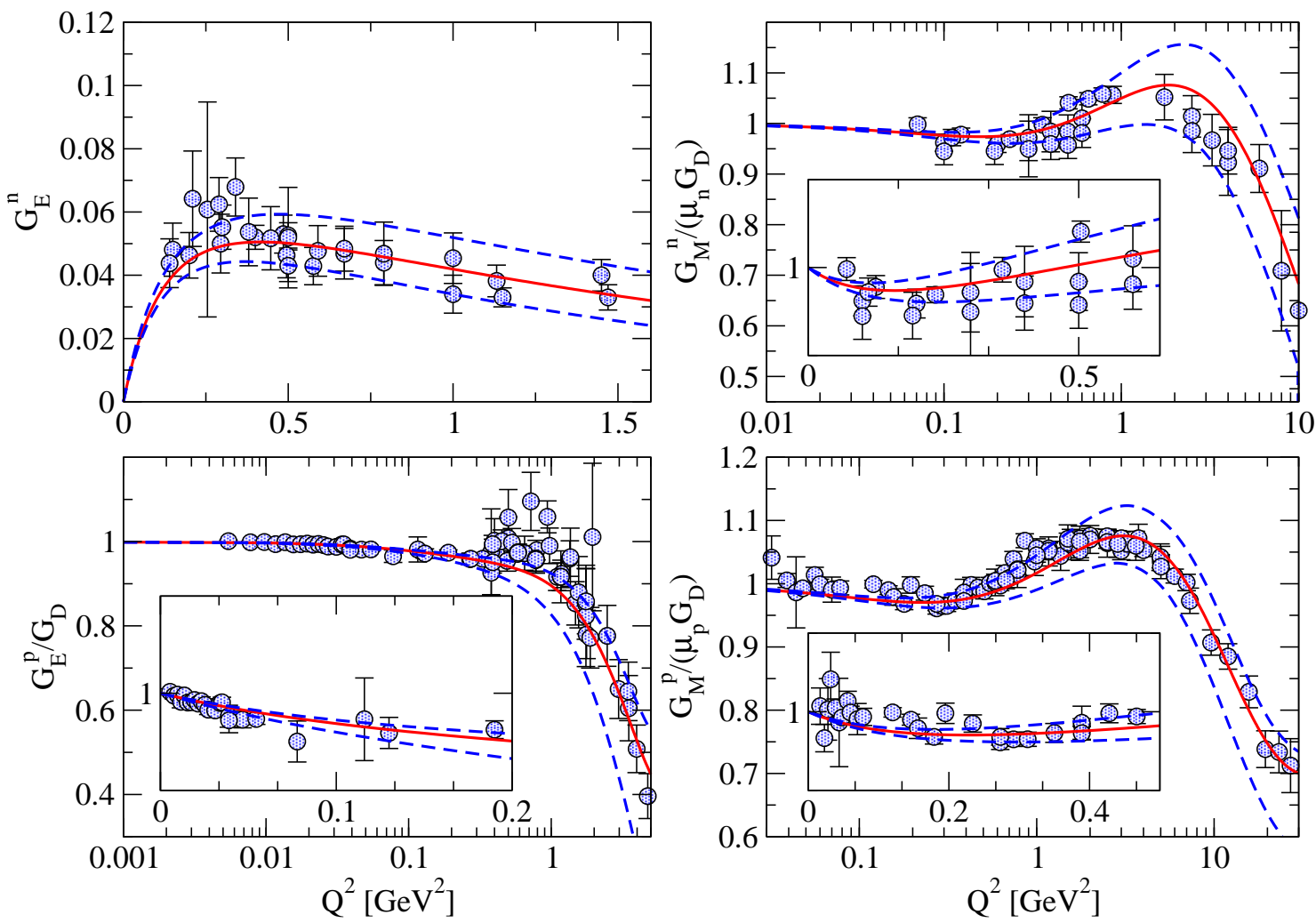

FIG. 4: The nucleon electromagnetic form factors for space-like momentum transfer in the SC approach. The solid line gives our best fit while the dashed lines indicate the error band obtained by the $1 \sigma$ deviation as discussed above.

In Fig. 4, we show the results in the SC approach for all four form factors in the space-like region compared to the world data. In general, we get a good description of all data within our error bands. For $G_{E}^{p}$ there is some inconsistency in the data points around $Q^{2} \approx 1 \mathrm{GeV}^{2}$. Our fit favors the lower data points in this region. As can be seen from the inset, the data at low momentum transfers are well described. In addition to the $\omega$ and the residual $\phi$, this fit has 2 more isoscalar poles $\left(M_{s_{1}} \approx 1.1 \mathrm{GeV}, M_{s_{2}} \approx 2.0 \mathrm{GeV}\right)$ and 5 isovector poles with masses ranging from 1 to $3 \mathrm{GeV}$. The heaviest poles in both channels are broad resonances (cf. Eq. (20) ) with width parameters ranging from $5 \mathrm{GeV}$ (isoscalar) to $19 \mathrm{GeV}$ (isovector). All other poles have zero widths. The fit has 17 free parameters and a total $\chi^{2} /$ dof of 1.8. The fit parameters are listed in detail in Table $\amalg$ in the Appendix. 
Note also that we do not obtain a pronounced bump-dip structure in $G_{E}^{n}$ as observed in Ref. [27]. However, all data for $G_{E}^{n}$ are described within our error band and the experimental error. We will come back to this bump-dip structure in Sec. VE and discuss the modifications in the spectral function required to produce this structure.

In Fig. 15, we show our fit results for the $\mathrm{SC}$ approach in the time-like region. As in the
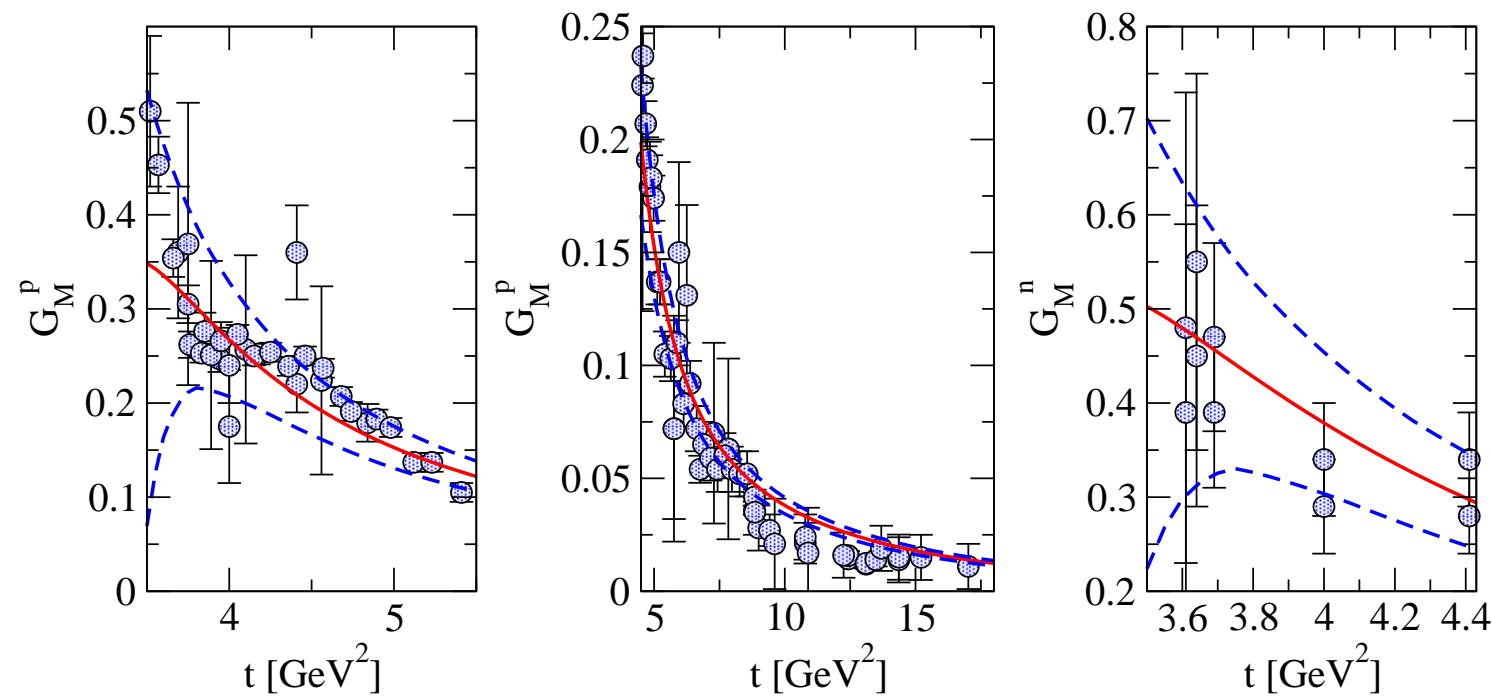

FIG. 5: The nucleon electromagnetic form factors for time-like momentum transfer in the SC approach (right panel: $G_{n}^{M}$, left panels: $G_{p}^{M}$ ). The proton data participate in the fit while the neutron data are a genuine prediction. The solid line gives our best fit while the dashed lines indicate the error band obtained by the $1 \sigma$ deviation as discussed above.

space-like region we get a good description of the world data within our error bands. Our best fit, however, cannot reproduce the strong rise of the data for $G_{M}^{p}$ near threshold. The data for $G_{M}^{n}$ are also well described. Note that the neutron data do not participate in the fit and the corresponding curves are therefore genuine prediction of the dispersion analysis based on data in the other channels.

\section{B. Explicit pQCD Continuum Approach}

In Fig. 6, we show our results in the explicit pQCD continuum approach for space-like momentum transfers compared to the world data. Again, we get a good description of all data within our error bands. In contrast to the superconvergence approach, our fit now favors somewhat larger values of $G_{E}^{p}$ in the region around $Q^{2} \approx 1 \mathrm{GeV}^{2}$. For $G_{E}^{n}$ the situation is the same as before: we describe all data within our error band and the experimental errors but see no pronounced bump-dip structure in the fits. In addition to the $\omega$ and the residual $\phi$, this fit has one more isoscalar pole $\left(M_{s_{1}} \approx 1.8 \mathrm{GeV}\right)$ and 3 isovector poles $\left(M_{v_{1}} \approx 1.0 \mathrm{GeV}\right.$, $M_{v_{2}} \approx 1.6 \mathrm{GeV}$, and $\left.M_{v_{3}} \approx 1.8 \mathrm{GeV}\right)$. Moreover, it contains an explicit pQCD continuum term, Eq. (21), as discussed above. This fit has 14 free parameters and a total $\chi^{2} /$ dof of 2.0. In general, we cannot get a satisfactory description of all data using fewer parameters. The parameters of this fit are given in detail in Tables III and IV in the Appendix.

In Fig. [7] we show the results in the time-like region. As before we describe the time-like data within our error band, but the errors increase strongly close to threshold. Our best fit 

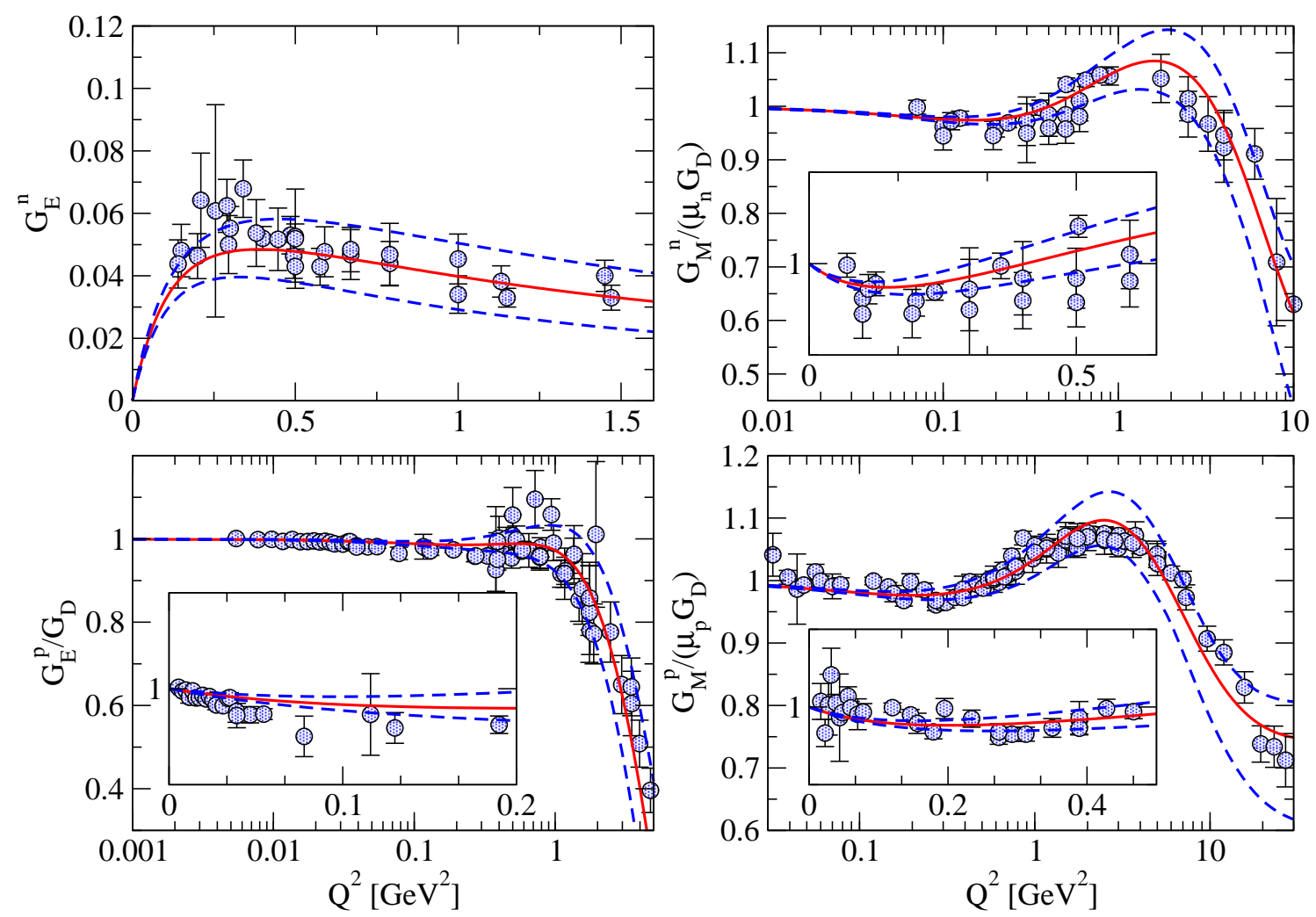

FIG. 6: The nucleon electromagnetic form factors for space-like momentum transfer with the explicit pQCD continuum. The solid line gives our best fit while the dashed lines indicate the error band obtained by the $1 \sigma$ deviation as discussed above.

turns over very close to threshold and cannot describe the two lowest data points. The error band is even larger for the neutron data which do not participate in the fit. But within the $1 \sigma$ band the neutron data are well described by this fit. Due to the strong increase of the $1 \sigma$ band, however, we can not make precise predictions for the time-like form factors close to threshold.

\section{Nucleon Radii and Coupling Constants}

In Table II we give the nucleon radii extracted from our fits in the $\mathrm{SC}$ and explicit pQCD approaches. The first number gives the value for our best fit, while the numbers in parentheses indicate the range from the $1 \sigma$ band. Our values are compared to the results of Ref. 23] and other recent determinations from low-momentum transfer data [52, 72, 73, 74, 75, 76].

The nucleon radii are generally in good agreement with other recent determinations using only low-momentum-transfer data given in the table. In particular, the squared neutron charge radius is in good agreement with the experimental value. In previous analyses [20, 23], this radius was constrained to the experimental value and not a prediction. Our result for the proton charge radius, however, is somewhat small. This was already the case in the earlier dispersion analyses of Refs. [20, 23]. We speculate that the reason for this discrepancy lies in inconsistencies in the data sets. In this type of global analysis all four form factors are 

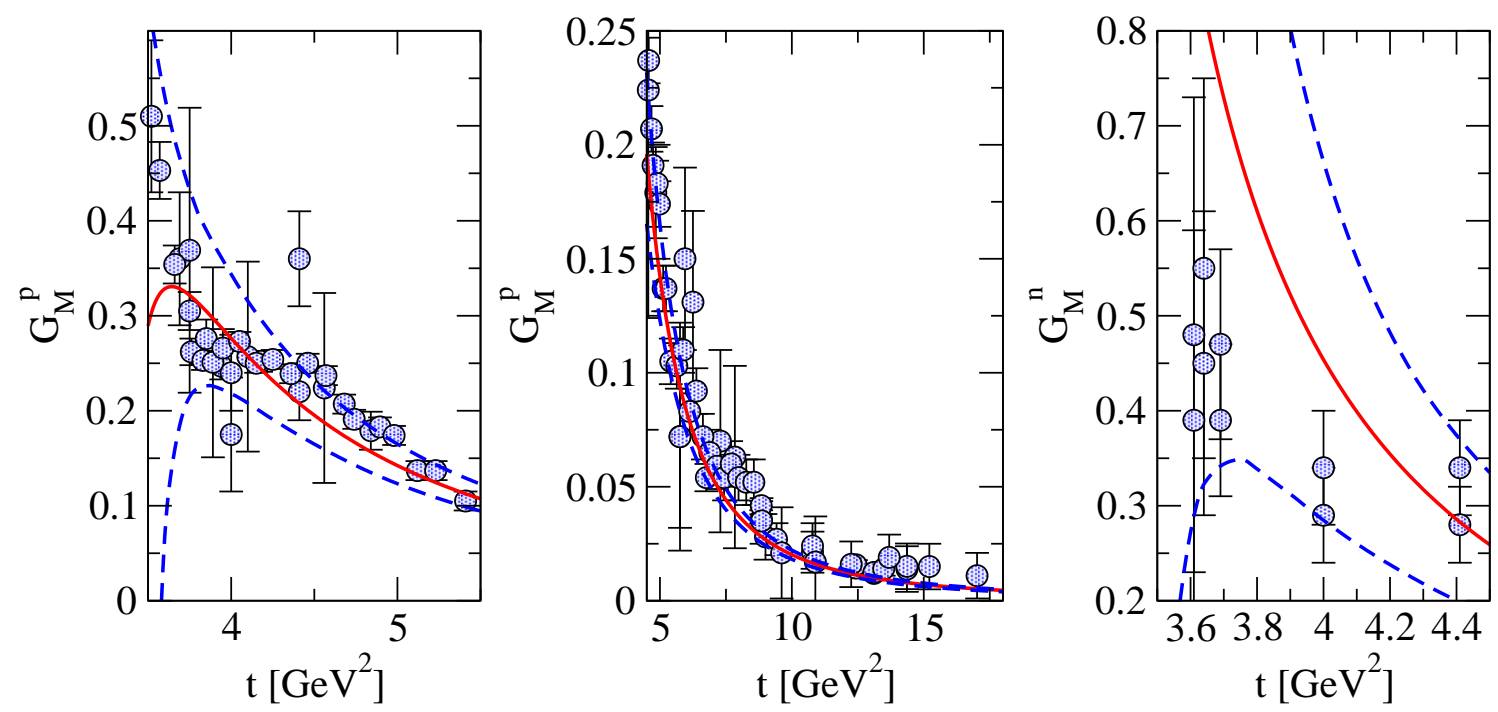

FIG. 7: The nucleon electromagnetic form factors for time-like momentum transfer with the explicit pQCD continuum (right panel: $G_{n}^{M}$, left panels: $G_{p}^{M}$ ). The proton data participate in the fit while the neutron data are a genuine prediction. The solid line gives our best fit while the dashed lines indicate the error band obtained by the $1 \sigma$ deviation as discussed above.

\begin{tabular}{|c|c|c|c|c|}
\hline & SC approach & explicit pQCD app. & Ref. [23] & recent determ. \\
\hline \hline$r_{E}^{p}[\mathrm{fm}]$ & $0.844(0.840 \ldots 0.852)$ & $0.830(0.822 \ldots 0.835)$ & 0.848 & $0.886(15)[72,73,74]$ \\
$r_{M}^{p}[\mathrm{fm}]$ & $0.854(0.849 \ldots 0.859)$ & $0.850(0.843 \ldots 0.852)$ & 0.857 & $0.855(35)[73,75]$ \\
$\left(r_{E}^{n}\right)^{2}\left[\mathrm{fm}^{2}\right]$ & $-0.117(-0.11 \ldots-0.128)$ & $-0.119(-0.108 \ldots-0.13)$ & -0.12 & $-0.115(4)[52]$ \\
$r_{M}^{n}[\mathrm{fm}]$ & $0.862(0.854 \ldots 0.871)$ & $0.863(0.859 \ldots 0.871)$ & 0.879 & $0.873(11)[76]$ \\
\hline
\end{tabular}

TABLE I: Nucleon radii extracted from our fits in the SC (2nd column) and explicit pQCD approaches (3rd column). The first number gives the value for our best fit, while the numbers in parentheses indicate the range from the $1 \sigma$ band. For comparison, we give the results of Ref. 23. (4th column) and other recent determinations from low-momentum transfer data 52, 72, 73, 74, 75, 76] (5th column).

analyzed simultaneously and both data at small and large momentum transfers enter. This can be an advantage or a disadvantage depending on the question at hand.

The discrepancy is not likely to be explained by $2 \gamma$ physics. In Ref. 77], it was shown that $2 \gamma$ exchange (including intermediate nucleons only) has a very tiny effect on the extraction of the proton radius from $e p$ scattering data. We have also performed various fits where the proton charge radius was constrained to values between 0.88 and $0.90 \mathrm{fm}$. The quality of these fits is not quite as good as for our best fits but still acceptable $\left(\chi^{2} /\right.$ dof $\left.\approx 2.6\right)$. The fits exhibit a pronounced bump-dip structure in $G_{E}^{p}$. However, they are on the low side of the data in the interval $Q^{2}=0.3 \ldots 0.8 \mathrm{GeV}^{2}$. These conclusions remain valid if we use a $2 \gamma$ corrected data basis for the proton form factor (including intermediate nucleons in the $2 \gamma$ exchange only) [78].

We have also extracted the $\omega N N$ couplings from our fit. The $\omega N N$ coupling constant is 
related to the residua via

$$
g_{\omega N N}^{i}=\frac{f_{\omega}}{M_{\omega}^{2}} a_{i}^{\omega}, \quad i=1,2
$$

where $f_{\omega}=17$ is the electromagnetic coupling of the $\omega$. The $\omega$ resonance couplings, e.g., play an important role in addressing the issue of isospin violation in the nucleon form factors [79]. While the vector residua of the $\omega$ are fixed relatively well by the fits, $a_{\omega}^{1}=0.60 \ldots 0.83 \mathrm{GeV}^{2}$, even the sign of the tensor residua can not be determined, $a_{\omega}^{2}=-0.13 \ldots 0.37 \mathrm{GeV}^{2}$. This leads to the following range for the $\omega N N$ coupling constants:

$$
g_{\omega N N}^{1}=16.7 \ldots 23.1, \quad \text { and } \quad g_{\omega N N}^{2}=-3.6 \ldots 10.3 .
$$

We have not extracted a coupling constant for the $\phi$ to the nucleon since the $\phi$ strength appears also in the $K \bar{K}$ and $\rho \pi$ continua and the interpretation of the residual $\phi$ pole strength is ambiguous.

\section{Inclusion of the preliminary CLAS data}

The CLAS collaboration at Jefferson Lab has recently taken new data for $G_{M}^{n}$ in the range $0.6 \leq Q^{2} \leq 5 \mathrm{GeV}^{2}[71]$. These data are still preliminary and were not included in the fits discussed above. In the this subsection, we present a fit in the explicit pQCD continuum approach where the preliminary CLAS data are included. In Fig. 8, we show our results for space-like momentum transfers compared to the published world data (blue circles) and the preliminary CLAS data (green triangles) 71]. Again, we get a good description of most data within our error bands. In the region $0.5 \mathrm{GeV}^{2} \lesssim Q^{2} \lesssim 1.0 \mathrm{GeV}^{2}$ the preliminary CLAS data differ significantly from most of the published world data. The reason for this discrepancy is not yet understood. Our fit prefers the CLAS data in this region. Some of the world data are even out of our $1 \sigma$ band. The description of the timelike data and the nucleon radii in this fit is of similar quality as for the fits described in subsections $V A$ and $V B$.

In addition to the $\omega$, this fit has two more isoscalar poles $\left(M_{s_{1}} \approx 1.1 \mathrm{GeV}\right.$ and $M_{s_{2}} \approx$ $1.4 \mathrm{GeV})$ and 3 isovector poles $\left(M_{v_{1}} \approx 1.0 \mathrm{GeV}, M_{v_{2}} \approx 1.6 \mathrm{GeV}\right.$, and $\left.M_{v_{3}} \approx 1.8 \mathrm{GeV}\right)$. Moreover, it contains an explicit pQCD continuum term, Eq. (21), as discussed above. This fit has 15 free parameters and a total $\chi^{2} /$ dof of 2.2 . The slight increase in the total $\chi^{2}$ compared to the similar fit in subsection $\mathrm{VB}$ is due to the inconsistency between the preliminary CLAS data and the older data in the region $0.5 \mathrm{GeV}^{2} \lesssim Q^{2} \lesssim 1.0 \mathrm{GeV}^{2}$. The parameters of this fit are given in detail in Tables $\nabla$ and VI in the Appendix.

\section{E. Pion Cloud of the Nucleon}

Friedrich and Walcher (FW) recently analysed the em nucleon form factors and performed various phenomenological fits [27]. Their fits showed a pronounced bump-dip structure in $G_{E}^{n}$ which they interpreted using an ansatz for the pion cloud based on the idea that the proton can be thought of as a virtual neutron-positively charged pion pair. They found a very long-range contribution to the charge distribution in the Breit frame extending out to about $2 \mathrm{fm}$ which they attributed to the pion cloud. While naively the pion Compton wave length is of this size, these findings are indeed surprising if compared with the "pion cloud" contribution due to the $2 \pi$ continuum contribution to the isovector spectral functions discussed in Sec. ஹIA. 

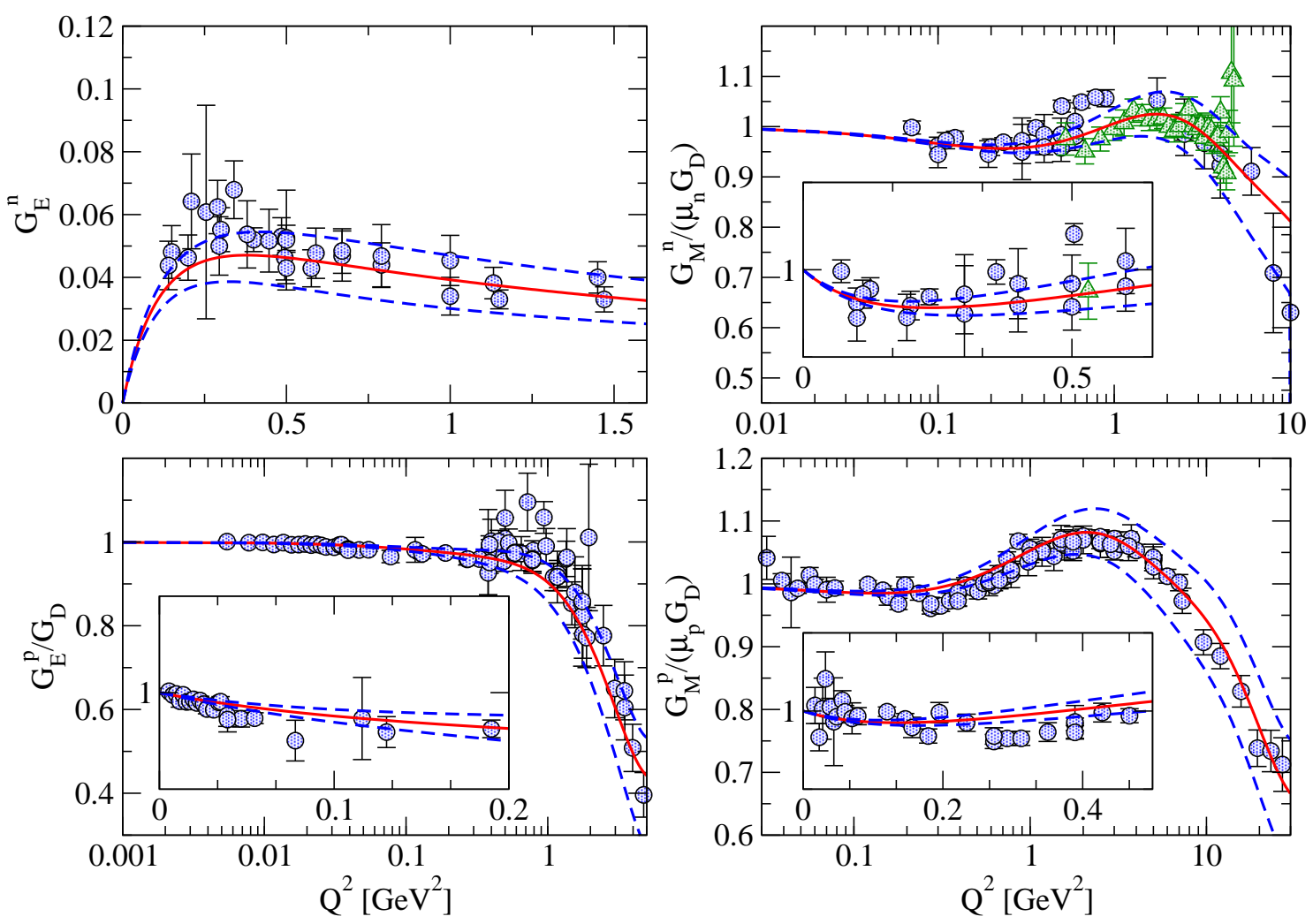

FIG. 8: The nucleon electromagnetic form factors for space-like momentum transfer with the explicit pQCD continuum. The blue circles indicate the published world data while the green triangles give the preliminary CLAS data for $G_{M}^{n}$ [1]. The solid line gives our best fit while the dashed lines indicate the error band obtained by the $1 \sigma$ deviation as discussed above.

As was shown by Hammer, Drechsel, and Meißner [28], the $2 \pi$ continuum contributions to the long-range part of the nucleon structure are much more confined in coordinate space and agree well with calculations in ChPT 47] and earlier (but less systematic) calculations based on chiral soliton models, see e.g. [80]. In the dispersion-theoretical framework, the longest-range part of the pion cloud contribution to the nucleon form factors is given by the $2 \pi$ continuum - the lowest-mass intermediate state including only pions.

As a consequence, it remains to be shown how the proposed long-range pion cloud can be reconciled with what is known from dispersion relations and ChPT. In order to clarify this issue, we have performed various fits in order to understand what structures in the spectral function are required to reproduce the bump in $G_{E}^{n}$. We find that the structure can only be reproduced if additional low-mass strength in the spectral function below $t \lesssim 1 \mathrm{GeV}^{2}$ is allowed beyond the $2 \pi, K \bar{K}$, and $\rho \pi$ continua and the $\omega$ pole. (See also Ref. [59] for some preliminary results on this question.) Since the spectral function is well understood in this region in terms of meson continua and vector meson dominance, such strength was explicitly excluded in the fits of Sec. D.

In Fig. 9, we show the results in the explicit pQCD approach with additional low-mass strength allowed. In this fit, all constraints were removed, the neutron charge form factor behaviour in the region of the bump-dip structure was enhanced by artificially lowering the error bars on the experimental data as seen by the fit. The results for all four form factors in the space-like region are compared to the world data. 

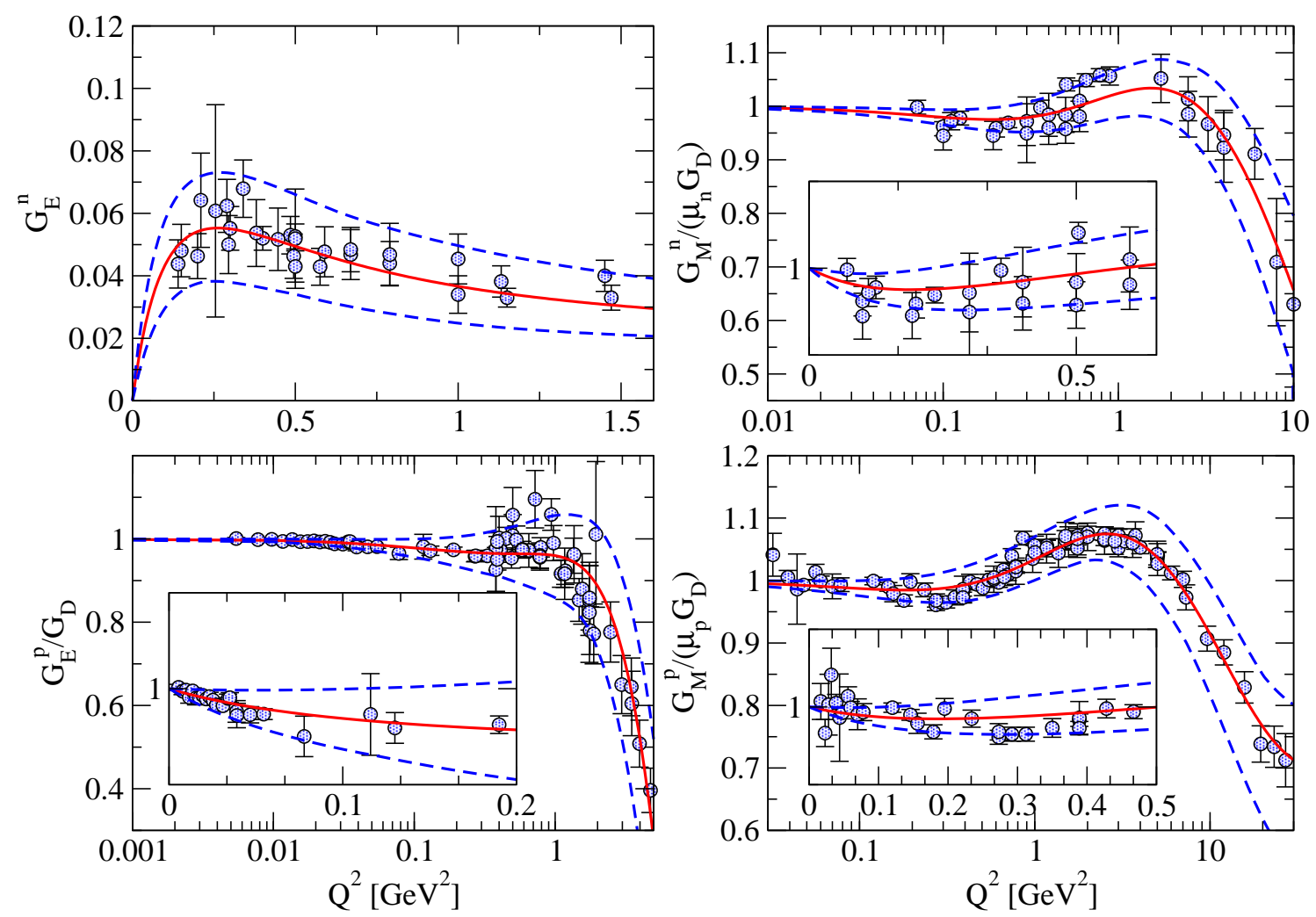

FIG. 9: The nucleon electromagnetic form factors for space-like momentum transfer with bump-dip structure for $G_{E}^{n}$. The solid line gives our best fit while the dashed lines indicate the error band obtained by the $1 \sigma$ deviation as discussed above.

In general, we get a good description of all data within our error bands. In particular, we obtain the desired bump-dip structure in $G_{E}^{n}$ at the cost of low-mass poles, which appear close to the $\omega$ mass in the isoscalar channel, and close to the 3-pion threshold in the isovector channel. The latter pole is weakly coupled. The behavior of the proton charge form factor around $Q^{2} \sim 1 \mathrm{GeV}^{2}$ is not resolved. The fit has 22 parameters and two effective poles (one isoscalar and one isovector) come out below $1 \mathrm{GeV}$. In addition to the $\omega$, this fit has 3 isoscalar poles and 4 isovector poles. One of the isovector poles is a broad resonance with a width parameter of order $13 \mathrm{GeV}$. The time-like data are not included in the fit. For the space-like data alone the total $\chi^{2} /$ dof is 0.9 . The parameters of this fit are listed in detail in Tables VII and VIII in the Appendix.

While this fit gives a good description of the space-like data, it is in contradiction to much of what is known about the structure of the spectral function below $1 \mathrm{GeV}$. Finally, we note again that the error bars of the data on $G_{E}^{n}$ in the region of the bump-dip structure had to be lowered artificially in order to obtain the desired structure. Taking it seriously requires to overcome the constraints imposed by unitarity and analyticity in the spectral function below $1 \mathrm{GeV}$. 


\section{SUMMARY \& OUTLOOK}

Dispersion theory simultaneously describes all four nucleon form factors over the whole range of momentum transfers in both the space-like and time-like regions. It allows for the inclusion of constraints from other physical processes, unitarity, and ChPT and therefore is an ideal tool to analyze the form factor data.

We have presented the results of our new form factor analysis. The spectral function has been improved compared to earlier analysis in various respects. (i) It contains the updated $2 \pi$ continuum [38], as well the $K \bar{K}$ [35, 36] and $\rho \pi$ continua 37] as independent input. (ii) The pQCD behavior of the form factors at large momentum transfer has been included in two different ways: using superconvergence relations and a broad resonance to mimick the QCD continuum (SC) and by including an explicit pQCD continuum term. Moreover, we have generated $1 \sigma$ error bands for all our fits by performing a Monte Carlo sampling of all fits with a total $\chi^{2} /$ dof in the interval $\left[\chi_{\min }^{2}, \chi_{\min }^{2}+1.04\right]$.

Our fits give a consistent description of the world data in the space-like and time-like regions. We find that some residual $\phi$ pole strength is still required in addition to the $K \bar{K}$ and $\rho \pi$ continua. The FENICE data for the neutron time-like form factors do not participate in the fit but are reproduced within the errors bands. This is an improvement over our earlier studies [21, 22]. The nucleon radii are generally in good agreement with other determinations with the exception of the proton charge radius which comes out smaller for our best fits. As discussed in subsection $\mathrm{VC}$ enforcing a larger radius $r_{E}^{p} \simeq 0.88 \mathrm{fm}$ leeds to acceptable fits with a slightly higher $\chi^{2}$. While the $\omega N N$ vector coupling constant is determined relatively well by the fits, even the sign of the tensor coupling constant can not be determined. We have also performed fits including the preliminary data for $G_{M}^{n}$ from the CLAS collaboration at Jefferson Lab 71]. For lower $Q^{2}$ these data are in conflict with earlier data, still our fits seem to prefer the trend set by the CLAS data. The bump-dip structure in $G_{E}^{n}$ advocated by Friedrich and Walcher [27] can only be obtained by artificially lowering the error bars of the data and allowing additional strength in the spectral function below $1 \mathrm{GeV}$.

There are two important extensions of this work. First, one could fit directly to the Coulomb-corrected cross section data, which would eliminate possible inconsistencies in the form factor data basis [75]. This could be further refined by consistently removing the full $2 \gamma$ exchange from the cross section data. The latter, however, remains a formidable challenge because of the contribution of the nucleon excited states.

\section{Acknowledgments}

We thank Bastian Kubis and Ingo Sick for useful discussions. We also thank John Arrington, Will Brooks and Richard Milner for informative communications and the CLAS collaboration for allowing us to show their data prior to publication. The work was supported in part by the EU I3HP "Study of Strongly Interacting Matter" under contract number RII3-CT-2004-506078 and the DFG through funds provided to the SFB/TR 16 "Subnuclear Structure of Matter". 


\section{APPENDIX A: FIT PARAMETERS}

In this Appendix, we give the values of the fit parameters for the various fits. Table II] contains the parameter values for the fit in the SC approach.

\begin{tabular}{|c|c|c|c|c|}
\hline Resonance & Mass $[\mathrm{GeV}]$ & $a_{1}\left[\mathrm{GeV}^{2}\right]$ & $a_{2}\left[\mathrm{GeV}^{2}\right]$ & $\Gamma[\mathrm{GeV}]$ \\
\hline \hline$\omega$ & 0.782 & 0.755960 & 0.370592 & - \\
\hline$\phi$ & 1.019 & -0.776537 & -2.913229 & - \\
\hline$s_{1}$ & 1.124860 & 0.902379 & 2.484859 & - \\
\hline$s_{2}$ & 2.019536 & 0.022798 & -0.130622 & 5.158635 \\
\hline \hline$v_{1}$ & 1.062128 & -0.127290 & -2.162533 & - \\
\hline$v_{2}$ & 1.300946 & -1.243412 & 3.704233 & - \\
\hline$v_{3}$ & 1.493630 & 4.191380 & -7.091021 & - \\
\hline$v_{4}$ & 1.668522 & -3.176013 & 3.723858 & - \\
\hline$v_{5}$ & 2.915451 & 0.048987 & 0.075965 & 19.088297 \\
\hline
\end{tabular}

TABLE II: Fit parameters in the SC approach. This fit has 17 free parameters and a total $\chi^{2} /$ dof of 1.8 .

In Table III we list the resonance parameters for the fit in the explicit pQCD approach. The parameters for the explicit pQCD term are given in Table IV.

\begin{tabular}{|c|c|c|c|}
\hline Resonance & Mass $[\mathrm{GeV}]$ & $a_{1}\left[\mathrm{GeV}^{2}\right]$ & $a_{2}\left[\mathrm{GeV}^{2}\right]$ \\
\hline \hline$\omega$ & 0.782 & 0.616384 & 0.114681 \\
\hline$\phi$ & 1.019 & 0.159562 & -0.329255 \\
\hline$s_{1}$ & 1.799639 & 0.128654 & 0.026174 \\
\hline \hline$v_{1}$ & 1.000000 & -0.309199 & -1.078695 \\
\hline$v_{2}$ & 1.627379 & 3.695960 & -4.301057 \\
\hline$v_{3}$ & 1.779245 & -3.693109 & 3.630255 \\
\hline
\end{tabular}

TABLE III: Resonance parameters for the fit with explicit pQCD continuum. This fit has 14 free parameters and a total $\chi^{2} /$ dof of 2.0 .

\begin{tabular}{|c|c|c|c|}
\hline$a_{1}^{s}$ & $a_{1}^{v}$ & $b_{1}\left[\mathrm{GeV}^{-2}\right]$ & $c_{1}\left[\mathrm{GeV}^{-1}\right]$ \\
\hline 0.002321 & -0.028391 & 0.152903 & 0.161871 \\
\hline \hline$a_{2}^{s}$ & $a_{2}^{v}$ & $b_{2}\left[\mathrm{GeV}^{-3}\right]$ & $c_{2}\left[\mathrm{GeV}^{-1}\right]$ \\
\hline-0.126598 & -0.011693 & 1.159998 & 1.150000 \\
\hline
\end{tabular}

TABLE IV: Parameters of the explicit pQCD term for the fit with explicit pQCD continuum. This fit has 14 free parameters and a total $\chi^{2} /$ dof of 2.0 .

In Table $\nabla$ we list the resonance parameters for the fit including the preliminary data for $G_{M}^{n}$ from the CLAS collaboration at Jefferson Lab 71] in the range $0.6 \mathrm{GeV}^{2} \lesssim Q^{2} \lesssim 5 \mathrm{GeV}^{2}$. The parameters for the explicit pQCD term for the fit including the preliminary CLAS data 


\begin{tabular}{|c|c|c|c|}
\hline Resonance & Mass $[\mathrm{GeV}]$ & $a_{1}\left[\mathrm{GeV}^{2}\right]$ & $a_{2}\left[\mathrm{GeV}^{2}\right]$ \\
\hline \hline$\omega$ & 0.782 & 0.669166 & -0.135957 \\
\hline$s_{1}$ & 1.045277 & -0.025807 & 0.001144 \\
\hline$s_{2}$ & 1.400423 & 0.261240 & -0.053588 \\
\hline \hline$v_{1}$ & 1.022008 & -0.279441 & -1.215307 \\
\hline$v_{2}$ & 1.644552 & 3.823047 & -4.561225 \\
\hline$v_{3}$ & 1.770845 & -3.849954 & 4.027035 \\
\hline
\end{tabular}

TABLE V: Resonance parameters for the fit including the preliminary CLAS data for $G_{M}^{n}$ [1]. This fit has 15 free parameters and a total $\chi^{2} /$ dof of 2.2 .

\begin{tabular}{|c|c|c|c|}
\hline$a_{1}^{s}$ & $a_{1}^{v}$ & $b_{1}\left[\mathrm{GeV}^{-2}\right]$ & $c_{1}\left[\mathrm{GeV}^{-1}\right]$ \\
\hline-0.000186 & -0.026941 & 0.219241 & 0.169695 \\
\hline \hline$a_{2}^{s}$ & $a_{2}^{v}$ & $b_{2}\left[\mathrm{GeV}^{-3}\right]$ & $c_{2}\left[\mathrm{GeV}^{-1}\right]$ \\
\hline 0.000527 & -0.001835 & 0.004155 & 0.106343 \\
\hline
\end{tabular}

TABLE VI: Parameters of the explicit pQCD term for the fit including the preliminary CLAS data [1] for $G_{M}^{n}$. This fit has 15 free parameters and a total $\chi^{2} /$ dof of 2.2 .

for $G_{M}^{n}$ are given in Table VI.

In Table VII we list the resonance parameters for the fit with the bump-dip structure and additional low-mass strength allowed. The parameters for the explicit pQCD term in

\begin{tabular}{|c|c|c|c|c|}
\hline Resonance & Mass $[\mathrm{GeV}]$ & $a_{1}\left[\mathrm{GeV}^{2}\right]$ & $a_{2}\left[\mathrm{GeV}^{2}\right]$ & $\Gamma[\mathrm{GeV}]$ \\
\hline \hline$\omega$ & 0.782 & -3.088199 & 1.516336 & - \\
\hline$s_{1}$ & 1.087524 & -9.309347 & 5.152311 & - \\
\hline$s_{2}$ & 0.857075 & 7.969599 & -3.102716 & - \\
\hline$s_{3}$ & 1.145783 & 5.332548 & -3.754331 & - \\
\hline \hline$v_{1}$ & 0.315028 & 0.002785 & -0.008642 & - \\
\hline$v_{2}$ & 1.523890 & -3.257202 & 3.767630 & - \\
\hline$v_{3}$ & 1.323997 & 2.770486 & -5.497436 & - \\
\hline$v_{4}$ & 2.834388 & 0.177584 & -0.011050 & 13.477161 \\
\hline
\end{tabular}

TABLE VII: Resonance parameters for the fit with the bump-dip structure. This fit has 22 free parameters and a total $\chi^{2} /$ dof of 0.9 (space-like data only). Note that $s_{2}$ and $v_{1}$ are the additional low-mass poles necessary to generate the bump-dip structure.

this fit are given in Table VIII 


\begin{tabular}{|l|l|l|l|}
\hline$a_{1}^{s}$ & $a_{1}^{v}$ & $b_{1}\left[\mathrm{GeV}^{-2}\right]$ & $c_{1}\left[\mathrm{GeV}^{-1}\right]$ \\
\hline-0.786259 & -0.320320 & 0.971368 & 1.235451 \\
\hline \hline$a_{2}^{s}$ & $a_{2}^{v}$ & $b_{2}\left[\mathrm{GeV}^{-3}\right]$ & $c_{2}\left[\mathrm{GeV}^{-1}\right]$ \\
\hline-0.000484 & 0.033410 & 0.091209 & 0.994702 \\
\hline
\end{tabular}

TABLE VIII: Parameters of the explicit pQCD term for the fit with bump-dip structure. This fit has 22 free parameters and a total $\chi^{2} /$ dof of 0.9 (space-like data only).

[1] H. Gao, Int. J. Mod. Phys. E 12, 1 (2003) [Erratum-ibid. E 12, 567 (2003)] arXiv:nucl-ex/0301002.

[2] C. E. Hyde-Wright and K. de Jager, Ann. Rev. Nucl. Part. Sci. 54, 217 (2004) arXiv:nucl-ex/0507001.

[3] Th. Udem et al., Phys. Rev. Lett. 79, 2646 (1997).

[4] D. H. Beck and B. R. Holstein, Int. J. Mod. Phys. E 10, 1 (2001) arXiv:hep-ph/0102053.

[5] M. Ostrick, Eur. Phys. J. A 28, s01, 81 (2006).

[6] R. Baldini and E. Pasqualucci, in Chiral Dynamics: Theory and Experiment, A.M. Bernstein and B.R. Holstein (eds.), Lect. Notes Phys. Vol. 452, Springer, Heidelberg, 1995.

[7] M. Ambrogiani et al. [E835 Collaboration], Phys. Rev. D 60, 032002 (1999).

[8] A. Antonelli et al., Phys. Lett. B 313, 283 (1993).

[9] A. Antonelli et al., Nucl. Phys. B 517, 3 (1998).

[10] T. K. Pedlar et al. [CLEO Collaboration], Phys. Rev. Lett. 95, 261803 (2005) arXiv:hep-ex/0510005.

[11] B. Aubert et al. [BABAR Collaboration], Phys. Rev. D 73, 012005 (2006) arXiv:hep-ex/0512023.

[12] M. Ablikim et al. [BES Collaboration], Phys. Lett. B 630, 14 (2005) arXiv:hep-ex/0506059.

[13] H. Fröhlich, W. Heitler and N. Kemmer, Proc. Roy. Soc. A 166, 155 (1938).

[14] G.F. Chew, R. Karplus, S. Gasiorowicz, and F. Zachariasen, Phys. Rev. 110, 265 (1958).

[15] P. Federbush, M.L. Goldberger, and S.B. Treiman, Phys. Rev. 112, 642 (1958).

[16] W.R. Frazer and J.R. Fulco, Phys. Rev. Lett. 2, 365 (1959).

[17] W. R. Frazer and J. R. Fulco, Phys. Rev. 117, 1609 (1960).

[18] G. Höhler et al., Nucl. Phys. B 114, 505 (1976).

[19] G. Höhler and E. Pietarinen, Nucl. Phys. B 95, 210 (1975).

[20] P. Mergell, U.-G. Meißner and D. Drechsel, Nucl. Phys. A 596, 367 (1996) arXiv:hep-ph/9506375.

[21] H.-W. Hammer, U.-G. Meißner and D. Drechsel, Phys. Lett. B 385, 343 (1996) arXiv:hep-ph/9604294.

[22] H.-W. Hammer, in Proc. of the $e^{+} e^{-}$Physics at Intermediate Energies Conference, ed. Diego Bettoni, eConf C010430, W08 (2001) arXiv:hep-ph/0105337.

[23] H.-W. Hammer and U.-G. Meißner, Eur. Phys. J. A 20, 469 (2004) arXiv:hep-ph/0312081.

[24] V. M. Braun, A. Lenz and M. Wittmann, Phys. Rev. D 73, 094019 (2006) arXiv:hep-ph/0604050.

[25] M. Göckeler, T. R. Hemmert, R. Horsley, D. Pleiter, P. E. L. Rakow, A. Schäfer and G. Schierholz [QCDSF Collaboration], Phys. Rev. D 71, 034508 (2005) arXiv:hep-lat/0303019. 
[26] C. Alexandrou, G. Koutsou, J. W. Negele and A. Tsapalis, Phys. Rev. D 74, 034508 (2006) arXiv:hep-lat/0605017.

[27] J. Friedrich and T. Walcher, Eur. Phys. J. A 17, 607 (2003) arXiv:hep-ph/0303054.

[28] H.-W. Hammer, D. Drechsel and U.-G. Meißner, Phys. Lett. B 586, 291 (2004) arXiv:hep-ph/0310240.

[29] J.J. Sakurai, Ann. Phys. (NY) 11, 1 (1960).

[30] G.J. Gounaris and J.J. Sakurai, Phys. Rev. Lett. 21, 244 (1968).

[31] M. Gari and W. Krümpelmann, Z. Phys. A 322, 689 (1985).

[32] E.L. Lomon, Phys. Rev. C 64, 035204 (2001) arXiv:nucl-th/0104039.

[33] S. Dubnicka, A.Z. Dubnickova and P. Weisenpacher, J. Phys. G 29, 405 (2003) arXiv:hep-ph/0208051.

[34] E. Tomasi-Gustafsson, F. Lacroix, C. Duterte and G. I. Gakh, Eur. Phys. J. A 24, 419 (2005) arXiv:nucl-th/0503001.

[35] H.-W. Hammer and M.J. Ramsey-Musolf, Phys. Rev. C 60, 045205 (1999) [Erratum-ibid. C 62, 049903 (2000)] arXiv:hep-ph/9812261.

[36] H.-W. Hammer and M.J. Ramsey-Musolf, Phys. Rev. C 60, 045204 (1999) [Erratum-ibid. C 62, 049902 (2000)] arXiv:hep-ph/9903367.

[37] U.-G. Meißner, V. Mull, J. Speth and J. W. van Orden, Phys. Lett. B 408, 381 (1997) arXiv:hep-ph/9701296.

[38] M.A. Belushkin, H.-W. Hammer and U.-G. Meißner, Phys. Lett. B 633, 507 (2006) arXiv:hep-ph/0510382.

[39] R.R. Akhmetshin et al. [CMD-2 Collaboration], arXiv:hep-ex/9904027, Phys. Lett. B 527, 161 (2002) arXiv:hep-ex/0112031, Phys. Lett. B 578, 285 (2004) arXiv:hep-ex/0308008.

[40] A. Aloisio et al. [KLOE Collaboration], Phys. Lett. B 606, 12 (2005) arXiv:hep-ex/0407048.

[41] M. N. Achasov et al., J. Exp. Theor. Phys. 101, 1053 (2005) arXiv:hep-ex/0506076.

[42] G. Höhler, Pion-Nucleon Scattering, Landolt-Börnstein Vol. I/9b, ed. H. Schopper, Springer, Berlin, 1983.

[43] G. Höhler and E. Pietarinen, Phys. Lett. B 53, 471 (1975).

[44] J. Gasser, M. E. Sainio and A. Svarc, Nucl. Phys. B 307, 779 (1988).

[45] U.-G. Meißner, Int. J. Mod. Phys. E 1, 561 (1992).

[46] V. Bernard, N. Kaiser and U.-G. Meißner, Nucl. Phys. A 611, 429 (1996) arXiv:hep-ph/9607428.

[47] N. Kaiser, Phys. Rev. C 68, 025202 (2003) arXiv:nucl-th/0302072.

[48] B. Kubis and U.-G. Meißner, Nucl. Phys. A 679, 698 (2001) arXiv:hep-ph/0007056.

[49] M.R. Schindler, J. Gegelia and S. Scherer, Eur. Phys. J. A 26, 1 (2005) arXiv:nucl-th/0509005.

[50] K. Holinde, Prog. Part. Nucl. Phys. 66, 311 (1996).

[51] S. Kopecky et al., Phys. Rev. Lett. 74, 2427 (1995).

[52] S. Kopecky, M. Krenn, P. Riehs, S. Steiner, J. A. Harvey, N. W. Hill, and M. Pernicka, Phys. Rev. C 56, 2229 (1997).

[53] S.J. Brodsky and G.P. Lepage, Phys. Rev. D 22, 2157 (1980).

[54] A. V. Belitsky, X. Ji and F. Yuan, Phys. Rev. Lett. 91, 092003 (2003) arXiv:hep-ph/0212351.

[55] J. P. Ralston and P. Jain, Phys. Rev. D 69, 053008 (2004) arXiv:hep-ph/0302043.

[56] E. Tomasi-Gustafsson and G. I. Gakh, Eur. Phys. J. A 26, 285 (2005) arXiv:hep-ph/0511077.

[57] I. Sabba-Stefanescu, J. Math. Phys. 21, 175 (1980).

[58] M. Galassi et al, "GNU Scientific Library Reference Manual (2nd Ed.)," ISBN 0954161734 
[59] H.-W. Hammer, Eur. Phys. J. A 28, s01, 49 (2006) arXiv:hep-ph/0602121.

[60] B. Plaster et al. [Jefferson Laboratory E93-038 Collaboration], Phys. Rev. C 73, 025205 (2006) arXiv:nucl-ex/0511025.

[61] D. I. Glazier et al., Eur. Phys. J. A 24, 101 (2005) arXiv:nucl-ex/0410026.

[62] G. Warren et al. [Jefferson Lab E93-026 Collaboration], Phys. Rev. Lett. 92, 042301 (2004) arXiv:nucl-ex/0308021.

[63] J. Bermuth et al., Phys. Lett. B 564, 199 (2003) arXiv:nucl-ex/0303015.

[64] V. Ziskin, "Measurement of the electric form factor of the neutron at low momentum transfers using a vector poralized deuterium gas target at BLAST," PhD thesis, MIT, April 2005.

http://blast.lns.mit.edu/PUBLIC_RESULTS/THESES/Ziskin.pdf

[65] B. Anderson et al. [Jefferson Lab E95-001 Collaboration], arXiv:nucl-ex/0605006.

[66] G. Bardin et al., Nucl. Phys. B 411, 3 (1994).

[67] A. Antonelli et al., Phys. Lett. B 334, 431 (1994).

[68] G. Bassompierre et al. [Mulhouse-Strasbourg-Turin Collaboration], Phys. Lett. B 68, 477 (1977).

[69] B. Delcourt et al., Phys. Lett. B 86, 395 (1979).

[70] D. Bisello et al. [DM2 Collaboration], Z. Phys. C 48, 23 (1990).

[71] J.D. Lachniet, "A high precision measurement of the neutron magnetic form factor using the CLAS detector," PhD thesis, Carnegie Mellon University, 2005.

[72] R. Rosenfelder, Phys. Lett. B 479, 381 (2000) arXiv:nucl-th/9912031.

[73] I. Sick, Phys. Lett. B 576, 62 (2003) arXiv:nucl-ex/0310008.

[74] K. Melnikov and T. van Ritbergen, Phys. Rev. Lett. 84, 1673 (2000) arXiv:hep-ph/9911277.

[75] I. Sick, private communication.

[76] G. Kubon et al., Phys. Lett. B 524, 26 (2002) arXiv:nucl-ex/0107016.

[77] P.G. Blunden and I. Sick, Phys. Rev. C 72, 057601 (2005) arXiv:nucl-th/0508037.

[78] J. Arrington, private communication.

[79] B. Kubis and R. Lewis, Phys. Rev. C 74, 015204 (2006) arXiv:nucl-th/0605006.]

[80] U.-G. Meißner, Phys. Rept. 161, 213 (1988). 